\title{
Long non-coding RNA NEAT1 confers oncogenic role in triple-negative breast cancer through modulating chemoresistance and cancer stemness
}

\author{
Vivian Yvonne Shin', Jiawei Chen", Isabella Wai -Yin Cheuk', Man-Ting Siu', Chi-Wang Ho ${ }^{1}$, Xian Wang ${ }^{2}$, \\ Hongchuan Jin $^{3}$ and Ava Kwong ${ }^{1,4,5}$
}

\begin{abstract}
Triple-negative breast cancer (TNBC) is a malignant subtype of breast cancer with the absence of targeted therapy, resulting in poor prognosis in patients. Chemotherapy remains the mainstay of treatment for TNBC; however, development of drug resistance is the main obstacle for successful treatments. In recent years, long non-coding RNA (IncRNA) has been implicated in multiple biological functions in various diseases, particularly cancers. Accumulating evidence suggested that IncRNA nuclear paraspeckle assembly transcript 1 (NEAT1) expression is dysregulated in many human cancers and thus is a useful prognostic marker for cancer patients. Nevertheless, the mechanism of how NEAT1 confers drug resistance in TNBC is still largely unknown. We performed IncRNA profiling by the LncRNA Profiler qPCR Array Kit in normal control (NC) and breast cancers (BC) blood samples and further validated in a larger cohort of samples by qRT-PCR. Gene expression level and localization were investigated by qRT-PCR, western blotting, and immunofluorescence staining. Flow cytometric analysis was carried out to detect cancer stem cells. Functional studies were performed both in vitro and in vivo xenograft model. Among 90 IncRNAs, NEAT1 was highly expressed in the blood samples of breast cancer patients than in NC. In particular, the expression of NEAT1 was higher in TNBC tissues than other subgroups. Functional studies revealed that NEAT1 conferred oncogenic role by regulating apoptosis and cell cycle progression in TNBC cells. We identified that knockdown of NEAT1 sensitized cells to chemotherapy, indicating the involvement in chemoresistance. Importantly, shNEAT1 reduced stem cell populations such as CD44+/CD24-, ALDH+, and SOX2+, implicating that NEAT1 was closely related to cancer stemness in TNBC. Our data highlighted the roles of NEAT1 chemoresistance and cancer stemness, suggesting that it could be used as a new clinical therapeutic target for treating TNBC patients especially those with drug resistance.
\end{abstract}

\section{Introduction}

Tremendous advances in human genomics during past decades have unravelled the transcriptional landscape that

\footnotetext{
Correspondence: Ava Kwong (akwong@asiabreastregistry.com)

${ }^{1}$ Department of Surgery, The University of Hong Kong, Pokfulam, Hong Kong

${ }^{2}$ Department of Medical Oncology, Sir Run Run Shaw Hospital, Medical School of Zhejiang University, Hangzhou, China

Full list of author information is available at the end of the article.

These authors contributed equally: Vivian Yvonne Shin, Jiawei Chen

Edited by E. Candi
}

is far more complex than what we originally expected. There are $>80 \%$ of the human genome transcribed ${ }^{1,2}$, however, $<2 \%$ of the transcribed genome codes for protein and the remaining genome consists of non-coding RNAs (ncRNAs). Extensive efforts have been made in this field and discovered $>3000$ ncRNAs with known functions to date $^{3}$. Long non-coding RNAs (lncRNAs) is a subgroup of ncRNAs characterized as transcripts $>200$ nucleotides that are not translated into protein which distinguish

\section{(c) The Author(s) 2019}

(c) (i) Open Access This article is licensed under a Creative Commons Attribution 4.0 International License, which permits use, sharing, adaptation, distribution and reproduction c. in any medium or format, as long as you give appropriate credit to the original author(s) and the source, provide a link to the Creative Commons license, and indicate if changes were made. The images or other third party material in this article are included in the article's Creative Commons license, unless indicated otherwise in a credit line to the material. If material is not included in the article's Creative Commons license and your intended use is not permitted by statutory regulation or exceeds the permitted use, you will need to obtain permission directly from the copyright holder. To view a copy of this license, visit http://creativecommons.org/licenses/by/4.0/. 


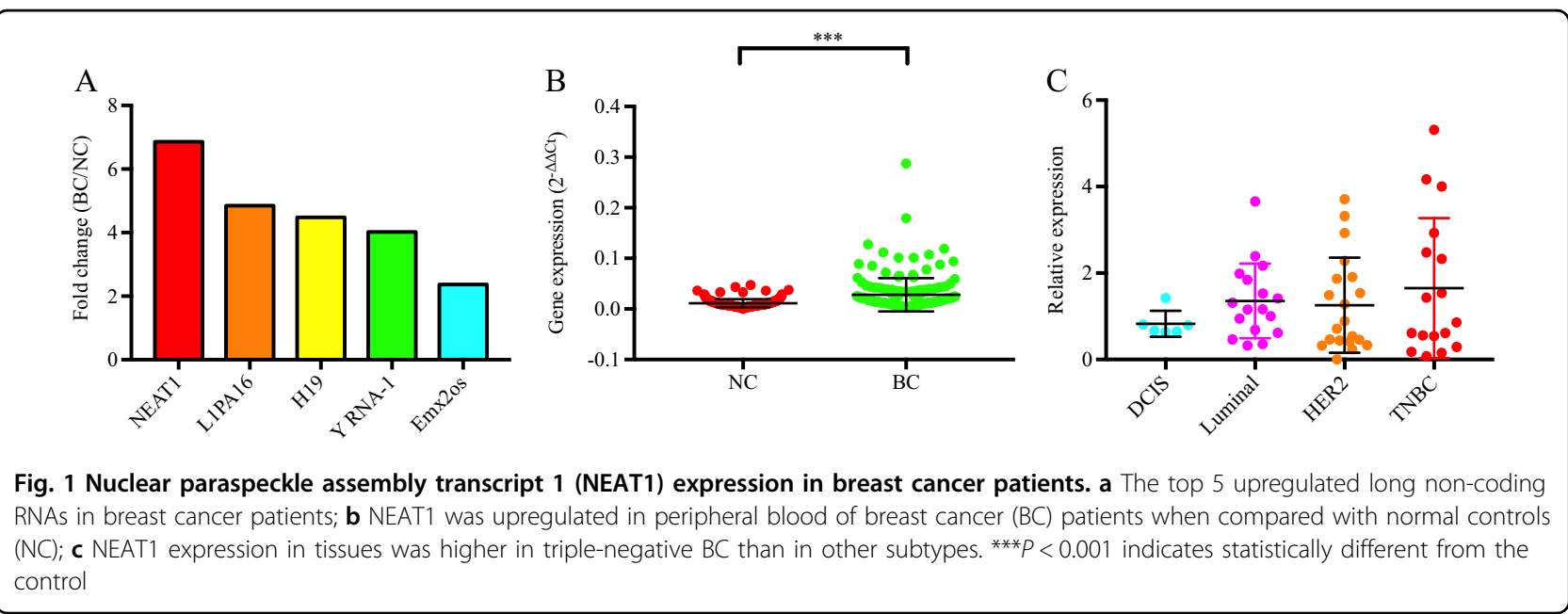

from other short ncRNAs such as microRNAs (miRNAs), short interfering RNAs (siRNAs), etc ${ }^{4}$. LncRNAs play critical roles in gene expression regulation on both transcriptional and posttranscriptional levels, resulting in a wide spectrum of biological processes including tumor initiation, growth and metastasis in different human diseases including cancers ${ }^{5-7}$. The nuclear-enriched abundant transcript 1 (NEAT1), which is a recently discovered essential component of nuclear paraspeckles ${ }^{8,9}$, is identified to be dysregulated in various solid cancers ${ }^{10,11}$. Under most circumstances, NEAT1 functions as a oncogene in different malignancies, such as lung cancer, oesophageal squamous cell carcinoma, laryngeal squamous cell carcinoma, ovarian cancer, colorectal cancer, hepatocellular carcinoma, prostate cancer, and glioma ${ }^{12-18}$. On the contrary, there are studies that reported the tumorsuppressive role of NEAT1 in other cancers, such as acute promyelocytic leukemia ${ }^{19}$. Also, it was reported to be regulated by $\mathrm{p} 53$ for tumor transformation suppression in pancreatic cancer $^{20}$ and low expression of NEAT1 was correlated with poor prognosis in colon, lung, and breast cancers $^{21}$.

Breast cancer is one of the most leading causes for cancer-related death among women worldwide, and the incidence rate is still increasing. Triple-negative breast cancer (TNBC), which lack the expression of estrogen receptor (ER), progesterone receptor (PR), and human epidermal growth factor receptor 2 (HER2), is an aggressive subtype of breast cancer with higher risk of early relapse and poor prognosis when compared with other subtypes ${ }^{22}$. By now, only limited studies reported on the action of NEAT1 in breast cancer, and others focused on its implication as a hypoxia-induced lncRNAs and led to accelerated cellular proliferation and increased tumorigenesis ${ }^{23}$. For example, NEAT1 promoted breast cancer growth by regulating miRNAs, such as miR- $548^{24}$ and miR- $448^{25}$. The FOXN3-NEAT1-SIN3A complex promoted epithelial-to- mesenchymal transition and invasion of breast cancer cells $^{26}$. In recent years, there has been convincing evidence of IncRNAs in the regulation of stem cell properties ${ }^{27-29}$; however, the mechanism involving cancer stem cells in relation to chemoresistance is still unclear. This study aims to examine the functional role of NEAT1 in stemness features and chemoresistance in TNBC.

\section{Results \\ High expression of NEAT1 in breast cancer patients}

Based on our microarray finding, we identified several dysregulated lncRNAs that are highly expressed in breast cancer patients. Among all, NEAT1 is the top upregulated lncRNA with a 6.86-fold increase (Fig. 1a). Further validation was performed in the circulation of 192 normal controls and 179 breast cancer patients by quantitative reverse transcription -PCR (qRT-PCR). The expression level of NEAT1 were significantly higher in breast cancer than in normal controls (Fig. 1b). We further evaluated the expression of NEAT1 in paired breast cancer tissues and stratified into ductal carcinoma in situ (DCIS), luminal, HER2, and TNBC subtypes. Results showed that NEAT1 expression was more prominent in TNBC than other subtypes (Fig. 1c), implicating an important role of NEAT1 in the carcinogenesis of TNBC. Since it is well known that NEAT1 has two isoforms (NEAT1-1 and NEAT1-2), we further investigated which specific isoform is the major form in TNBC. qRT-PCR result showed that there was no significant difference in the expression of NEAT1-2 among subtypes including DCIS, luminal, HER2 and TNBC, suggesting that NEAT1-1 is the major form in TNBC (Supplementary Fig 1).

\section{NEAT1 is required for G1-phase cell cycle arrest and apoptosis \\ We developed NEAT1 stable knockdown in MDA-MB-231 and its cisplatin/taxol resistance clone,}




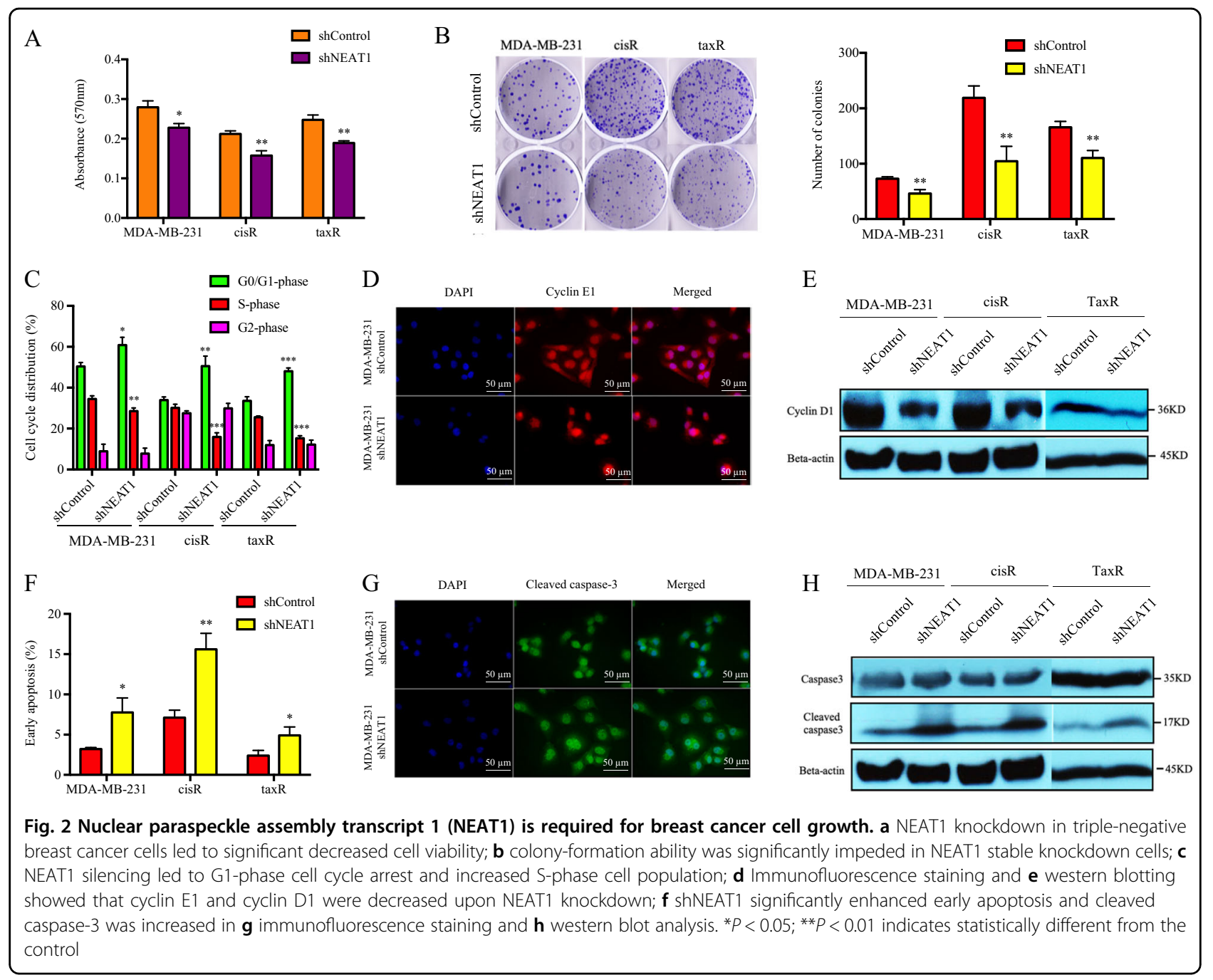

MDA-MB-231/cisR/taxR, to study the tumor-promoting effect of NEAT1 in TNBC. Significant inhibition of cell proliferation and colony formation was observed in shNEAT1 in both MDA-MB-231 and cisR/taxR (Fig. 2a, b). To understand the functional mechanism of growth inhibition, Annexin $\mathrm{V}$ binding assay was performed to detect apoptosis in shNEAT1 cells. Cell cycle analysis revealed a G1-phase arrest and increased $\mathrm{S}$-phase cell population in all shNEAT1 cells (Fig. 2c). Knockdown of NEAT1 reduced the expression of cyclin E1 and D1 (Fig. 2d, e), indicating that NEAT1 is required in cell cycle progression. Besides, early apoptosis was significantly increased in shNEAT1 cells (Fig. 2f), which was concomitant with increased cleaved caspase-3 by immunofluorescence staining and western blot analysis (Fig. 2g, h). All these data showed that blocking of NEAT1 had a more prominent effect on cell proliferation and apoptosis in cisR and taxR cells when compared with parental cells.

\section{NEAT1 mediated drug resistance and cancer stemness}

As NEAT1 expression is upregulated in cisR and taxR compared with its parental cells (Fig. 3a), suggesting its role in drug resistance, we further investigated the effect of NEAT1 on drug sensitivity. NEAT1 knockdown further decreased cell growth in cisplatin- or taxol-treated cells, demonstrating a synergistic effect of the combination of shNEAT1 and chemotherapeutic drugs in breast cancer (Fig. 3b). qRT-PCR results identified that drug transporter genes, such as ATP7A and ATP7B, were downregulated in shNEAT1 cells (Fig. 3c, d). Interestingly, a stemness marker, SOX2, was also downregulated in shNEAT1 cells (Fig. 3c, d). In single-cell clonogenic assays, significant fewer number of colonies and smaller colony size were observed in shNEAT1 cells (Fig. 4a), suggesting an impeded self-renewal ability. Next, flow cytometric analysis was used to quantify the cancer stem cell markerpositive cell population. CD44+/CD24-, ALDH + , and $\mathrm{SOX} 2+$ cell population decreased significantly in 

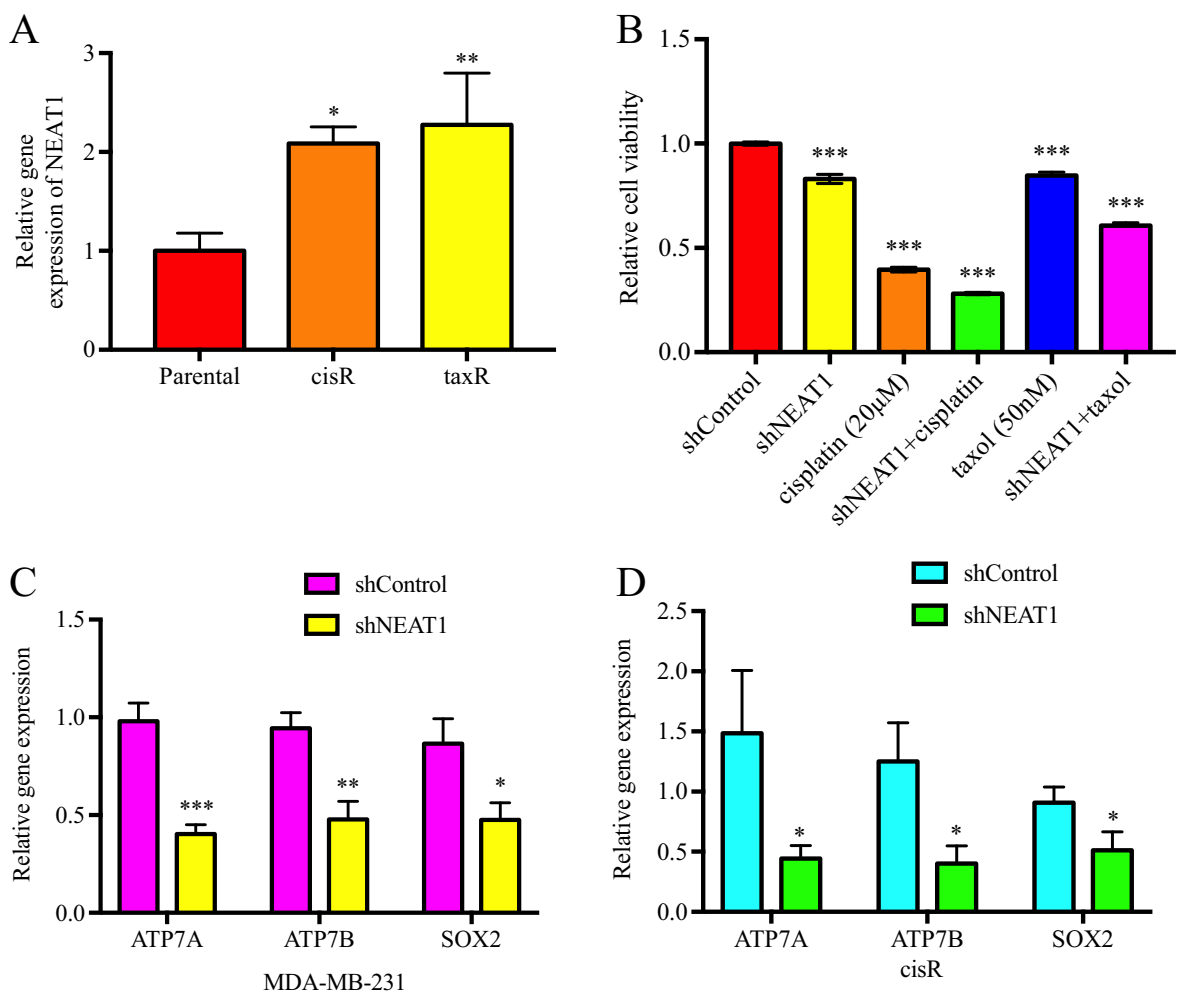

Fig. 3 Nuclear paraspeckle assembly transcript 1 (NEAT1) played important role in drug resistance. a NEAT1 expression was upregulated in cisplatin (cisR) and taxol (taxR) resistant cells; b NEAT1 knockdown had synergistic effect with chemotherapeutic drugs by inhibiting cancer cells growth; $\mathbf{c}$, d ATP7A/7B and SOX2 expression were decreased in shNEAT1 cells. ${ }^{*} P<0.05$; ${ }^{*} P<0.01 ;{ }^{* *} P<0.001$ indicates statistically different from the control

shNEAT1 cells (Fig. 4b-d) and SOX2 protein level was also downregulated by the loss of NEAT1 (Fig. 4e). These data illustrated that NEAT1 regulated cancer cell markers to regulate cell proliferation.

\section{NEAT1 silencing inhibited tumor growth in vivo}

To determine the effect of NEAT1 on breast cancer tumorigenicity in vivo, NOD-SCID mouse xenograft tumor model was used. shNEAT1 cells were injected subcutaneously into mammary fat pad of mice. Macroscopic observation of the injected mice revealed that tumor volume was smaller in shNEAT1-treated mice than in the shControl-treated mice (Fig. 5a, b). Meanwhile, aldehyde dehydrogenase (ALDH) and SOX2 expression levels were significantly lower in the shNEAT1-treated tumors compared with the shControl tumors (Fig. 5c, d). To further elucidate the role of NEAT1 in chemoresistance in in vivo model, cisplatin was administered locally in shControl- and shNEAT1-treated mice. Result showed that the tumor volume in the shNEAT1+cisplatin group was smaller than in the shNEAT1-treated group, indicating that NEAT1 suppression enhanced cisplatin sensitivity in breast cancer (Fig. 5e, f). Taken together, our results suggested that NEAT1 contributed in tumorigenesis of TNBC through regulating cancer stemness and chemoresistance.

\section{Discussion}

LncRNAs has long been considered as "transcriptional noise" as they do not code for proteins ${ }^{30}$. Currently, lncRNAs are now accepted as important regulators in cancer development and different biological processes such as chromatin remodeling and transcriptional and posttranscriptional regulation ${ }^{30}$. Enormous evidence showed that NEAT1 upregulation contributed to the progression of many malignancies ${ }^{31}$. A pan-cancer lncRNA analysis has shown that NEAT1 is overexpressed in tumor tissues, including bladder urothelial carcinoma, cervical squamous cell carcinoma, cholangiocarcinoma, colon carcinoma, esophageal carcinoma, head and neck squamous cell carcinoma, kidney clear cell carcinoma, hepatocellular carcinoma, pancreatic cancer, prostate cancer, gastric cancer, and thyroid cancer $^{32}$. On the contrary, only few reports suggested the tumor-suppressive role of NEAT1, such as leukemia ${ }^{19}$. In breast cancer, high expression of NEAT1 was correlated with poor overall survival of ER+ breast cancer patients. Besides, interaction of FOXN3 with 


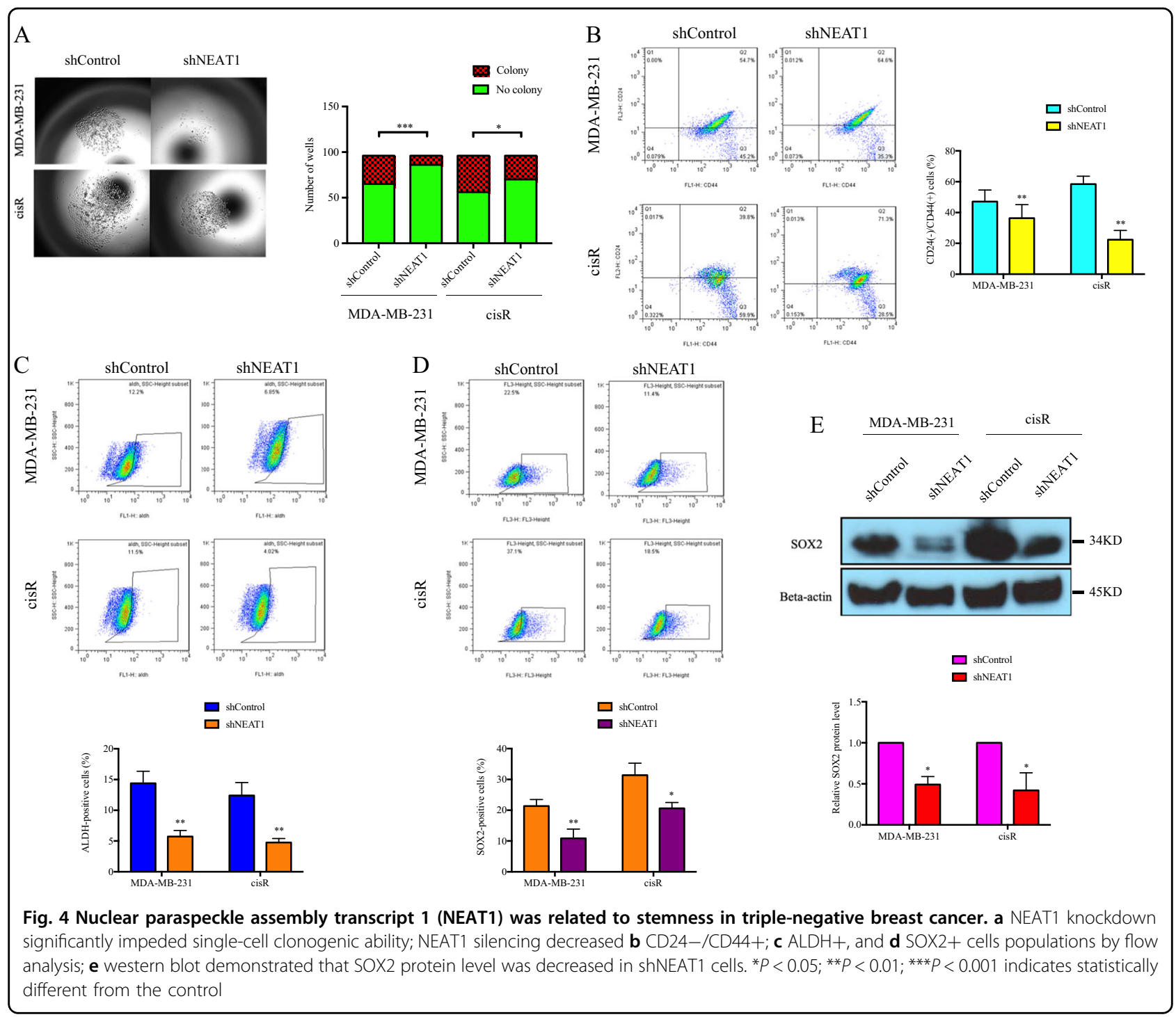

NEAT1/SIN3A showed to repress GATA3 in breast cancer metastasis ${ }^{26}$. In addition, functions of NEAT1 has been implicated in cell growth, migration, and invasion and regulated miR-488 and ZEB1 ${ }^{25}$. Patients with high NEAT1 expression in tumors correlated with poor survival $^{23}$. Hypoxia-inducible factor- 2 caused aggregation of nuclear paraspeckles in hypoxia condition, which induced pro-tumorigenic in breast cancer. Although recent studies revealed the crucial roles for lncRNAs in the breast cancer carcinogenesis ${ }^{24,26,33}$, the exact molecular mechanism underlying breast cancer is still unclear especially in TNBC. Strikingly, some studies revealed that NEAT1 could be detected in the circulation and was dysregulated in peripheral blood mononuclear cells in several cancers; these suggested that NEAT1 is a potential diagnostic and prognostic biomarker in cancers $^{34,35}$. In our study, we identified that NEAT1 expression was upregulated in the circulation of breast cancer patients and remarkably discriminated from normal controls. We are the first to report that NEAT 1 is upregulated in TNBC and expand our understanding on the role of NEAT1 in this malignant subtype of breast cancer. The inhibitory effect of NEAT1 knockdown caused reduction in cell proliferation, colony-forming ability, induction of apoptosis, and cell cycle arrest. This was also seen in ovarian cancer that NEAT1 suppressed apoptosis and increased S-phase cell population through regulating $\mathrm{Bcl} 2$ that is mediated by miR-34a-5 $\mathrm{p}^{36}$. In pancreatic cancer, NEAT1 facilitated its oncogenicity by directly binding to miR-506-3p, the miRNA that has been reported as a tumor suppressor in diverse cancers $^{37}$. Similar findings that NEAT1 functioned as tumor suppressor were reported in other cancers such as nonsmall cell lung cancer ${ }^{38}$, hepatocellular carcinoma ${ }^{39}$, and glioma ${ }^{40}$. All these findings put forward the dual action of NEAT1 in carcinogenesis. 


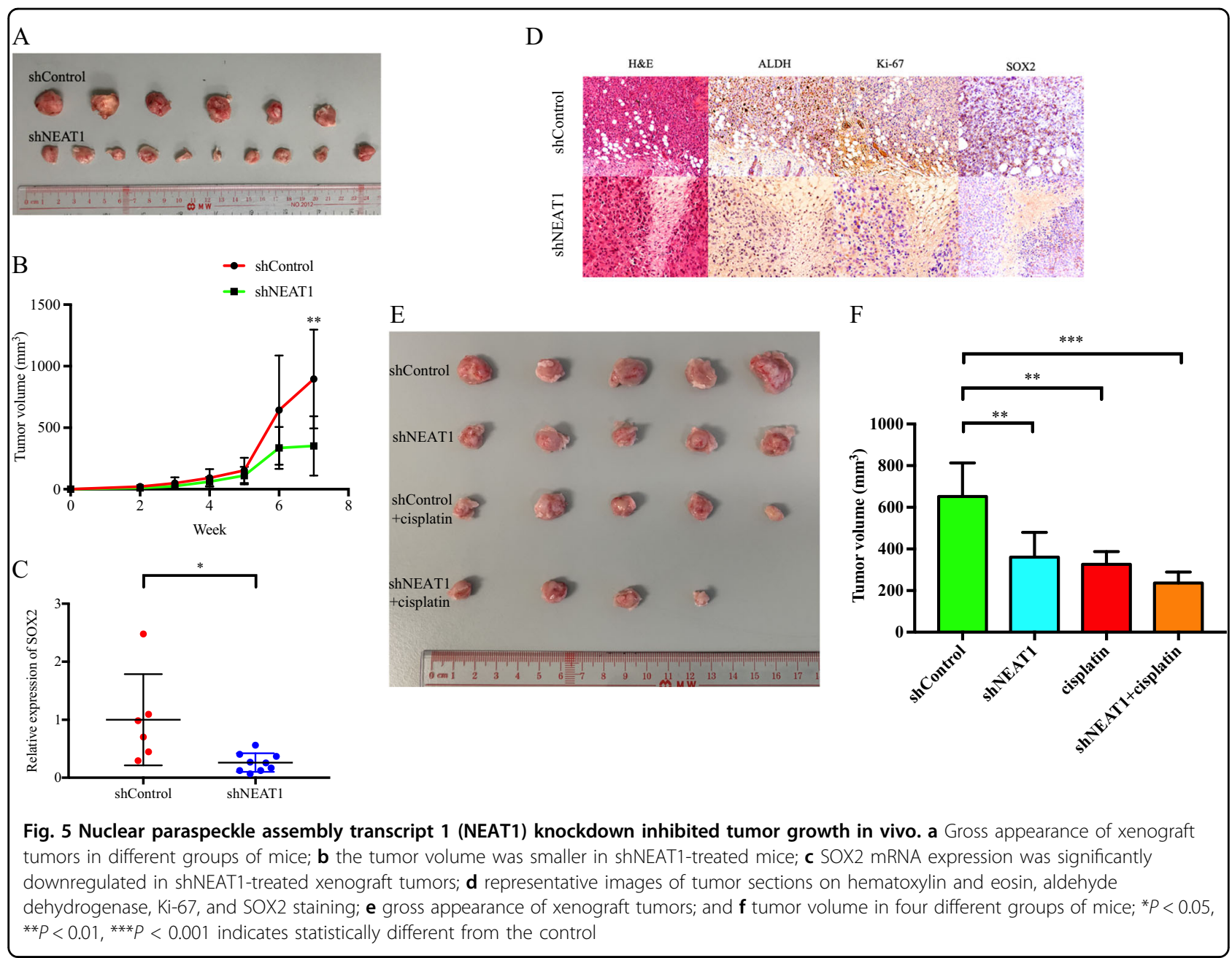

In concordance with our findings, there was evidence highlighting the involvement of NEAT1 in chemoresistance. In ovarian cancer, NEAT1 contributed to paclitaxel resistance partly through upregulating ZEB1 expression by sponging miR-194 ${ }^{41}$. In cisplatin-resistant gastric cancer cells, NEAT1 expression was significantly upregulated accompanied with the induction of P-glycoprotein, which is a multidrug resistance-associated protein $^{42}$. NEAT1 is also reported to contribute to docetaxel resistance through inducing RET expression by sponging miR$34 \mathrm{a}$ in prostate cancer ${ }^{43}$. In nasopharyngeal carcinoma, NEAT1 enhanced the cisplatin resistance by targeting Rsf- 1 and Ras-mitogen-associated protein kinase signaling pathway ${ }^{44}$. We revealed that the combination of chemodrug (cisplatin or taxol) and NEAT1 knockdown synergistically inhibited drug sensitivity when compared with cisplatin or taxol alone. Overexpression of NEAT1 in cisplatin- and taxol-resistant cells indicated its role chemoresistance in TNBC. Downregulation of drug transporter genes in shNEAT1 cells suggested a potential
NEAT1-mediated chemoresistance mechanism. During the past decade, very few studies delineated on the role of lncRNAs in stem cell biology ${ }^{45-47}$. Silencing of NEAT1 reduced cancer stem cell-like properties in lung cancer stem cells and CTR1 expression was negatively correlated with NEAT1 expression ${ }^{48}$. Furthermore, NEAT1 was upregulated in glioma stem cells, and inhibition of NEAT1 retarded cell proliferation, migration, and inva$\operatorname{sion}^{49}$. Lo et al. reported that in DCIS, NEAT1 was functionally required for maintaining stemness such as anchorage-independent growth in soft agar, self-renewal ability, and sphere-formation assay ${ }^{50}$. In conclusion, we found that NEAT1 was overexpressed in the circulation of breast cancer, especially TNBC. The tumor-promoting effect was due to dysregulated apoptosis and cell cycle. We identified for the first time that NEAT1 is associated with chemoresistance and cancer stem cell property in TNBC. These findings provide evidence that NEAT1 is a potential therapeutic target to overcome chemoresistance for treating TNBC. 


\section{Materials and Methods}

\section{Clinical specimen}

Breast cancer patients were recruited through Queen Mary Hospital, Tung Wah Hospital, and Hong Kong Sanatorium and Hospital through the Hong Kong Hereditary Breast Cancer Family Registry. This study was approved by Institutional Review Board of the University of Hong Kong (UW 15-441). All participants of this study including breast cancer patients and normal controls without personal history of cancers have agreed and signed the consent form. Blood samples from 179 breast cancer patients and 192 normal controls were included and the patients' demographic characteristics such as age, histological type, staging, metastasis, and subtypes are listed in Table 1.

\section{LncRNA expression profiling}

LncProfilers $^{\mathrm{TM}}$ qPCR Array Kit (System Biosciences, CA, USA) containing 90 lncRNAs was used to compare the lncRNA expression levels in breast cancer patients and

Table 1 Clinical characteristics of breast cancer patients

\begin{tabular}{|c|c|}
\hline & Breast cancer $(n=179)$ \\
\hline Age [years; mean (SD)] & $56.7(11.7)$ \\
\hline \multicolumn{2}{|l|}{ Histological type } \\
\hline DCIS & 16 \\
\hline IDC & 146 \\
\hline ILC & 5 \\
\hline Others & 12 \\
\hline Bilateral cancer & 5 \\
\hline \multicolumn{2}{|l|}{ Distant metastasis } \\
\hline Before Surgery & 1 \\
\hline After Surgery & 11 \\
\hline \multicolumn{2}{|l|}{ Stage } \\
\hline 0 & 26 \\
\hline I & 75 \\
\hline$\|$ & 55 \\
\hline III & 23 \\
\hline IV & 1 \\
\hline \multicolumn{2}{|l|}{ Subtypes } \\
\hline Luminal & 154 \\
\hline HER2 & 17 \\
\hline TNBC & 11 \\
\hline NA & 4 \\
\hline
\end{tabular}

DCIS ductal carcinoma in situ, IDC invasive ductal carcinoma, ILC invasive lobular carcinoma, HER2 human epidermal growth factor receptor 2, TNBC triple-negative breast cancer, NA not available normal controls. RNA from peripheral blood from five normal controls and five breast cancer patients were isolated using the QIAamp RNA Blood Mini Kit (Qiagen, CA, USA). After total RNA was extracted, lncRNAs were tagged with polyA tail and annealed to adaptor using the LncProfilers $^{\mathrm{Tm}}$ qPCR Array Kits. After the conversion of cDNA, qPCR assays were conducted. The relative amount of each lncRNA in normal controls compared to that in breast cancer was described using the $2^{-\Delta \Delta C T}$ method.

\section{RNA extraction and qRT-PCR}

Total RNA was extracted by using the QIAamp RNA Blood Mini Kit according to the manufacturer's standard instruction. Briefly, QIAzol Lysis Reagent was added to peripheral blood sample. After vortex homogenization, chloroform was used for separation followed by absolute ethanol to precipitate RNA. The eluted RNA concentration was quantified by NanoDrop 1000 (Thermo Scientific, DE, USA). Reverse transcription was performed by the High Capacity cDNA Reverse Transcription Kit (Thermo Fisher Scientific, CA, USA). LightCycler $^{\circledR} 480$ SYBR Green I Master (Roche, OR, USA) was used in the Roche LightCycler ${ }^{\circledR} 480$ System to perform qRT-PCR.

\section{Cell culture and transfection}

The metastatic TNBC cell line MDA-MB-231 $\left(\mathrm{ATCC}^{\circledR} \mathrm{HTB}^{-26^{\mathrm{TM}}}\right.$ ) was purchased from the American Type Culture Collection and cultured in RPMI 1640 medium (Invitrogen, Grand Island, USA) supplemented with $10 \%$ heat-inactivated fetal bovine serum. cisR or taxR sub-line of MDA-MB-231 cells were established by chronic treatment with cisplatin- or taxol-supplemented (Sigma-Aldrich, MO, USA) medium. The $\mathrm{IC}_{50}$ of cisplatin were 23.56 and $126.5 \mu \mathrm{M}$ in MDA-MB-231 and cisR cells (Supplementary Fig 2A), while the $\mathrm{IC}_{50}$ of taxol were 57.63 and $544.2 \mathrm{nM}$ in MDA-MB-231 and taxR cells (Supplementary Fig 2B), confirming the resistance characteristics of these two in-house developed cell lines. To develop stable negative control or NEAT1 knockdown clones, cells were transfected with negative control shRNA or NEAT1 shRNA expression vector with Lipofectamine 3000 Reagent (Thermo Fisher Scientific, CA, USA). The shRNA duplexes designed against NEAT1 (Gene ID: 283131) with the target sequence (CATGGACCGTGGTTTGTTACT) synthesized by GenePharma Company (Shanghai, China) were incorporated into the pGPU6/GFP/NeoshRNA vector to get pGPU6/GFP/Neo-shRNA-NEAT1. Cells were transfected with $1 \mu \mathrm{g}$ shRNA expression plasmids and were selected with $1 \mathrm{mg} / \mathrm{ml}$ Geneticin (Thermo Fisher Scientific, CA, USA) starting from 3 days after transfection. 


\section{Cell proliferation assay}

Thiazolyl blue tetrazolium bromide (MTT)-based assay was used to quantify cell viability after 3 days of cisplatin or taxol treatment. Briefly, $5 \times 10^{3}$ cells were seeded onto a 96-well plate and the medium was replaced with complete medium supplemented with 3\% MTT and incubated for $2 \mathrm{~h}$. Then the intracellular purple formazan crystal products were dissolved in $100 \mu \mathrm{l}$ of dimethyl sulfoxide (Sigma-Aldrich, MO, USA) followed by the colorimetric product measured by Multiskan ${ }^{\mathrm{Tm}}$ FC Microplate Photometer (Thermo Fisher Scientific, CA, USA) at $570 \mathrm{~nm}$.

\section{Apoptosis assay}

Fluorescein isothiocyanate (FITC) Annexin V Apoptosis Detection Kit (BD Biosciences, USA) was used to detect the apoptotic cell population according to manufacturer's standard protocol. Briefly, cells were collected and suspended in $1 \times$ Annexin $\mathrm{V}$ binding buffer at $1 \times 10^{6}$ cells $/ \mathrm{ml}$ density. In all, $100 \mu \mathrm{l}$ of resuspended cells were stained with $5 \mu \mathrm{l}$ of FITC Annexin $\mathrm{V}$ and $5 \mu \mathrm{l}$ of propidium iodide (PI). The mixture was vortexed at room temperature in dark for $15 \mathrm{~min}$. Then the cells were washed and resuspended in $400 \mu \mathrm{l}$ of $1 \times$ Annexin V-binding buffer and analyzed by BD FACSCalibur. At least 10,000 gated cells were counted in each sample for analysis calculation.

\section{Cell cycle analysis}

To determine the effect of NEAT1 knockdown on cell cycle distribution, cellular DNA contents were stained with PI for flow cytometric analysis. Briefly, cells were collected, washed with chilled phosphate-buffered saline (PBS), and fixed with ice-cold $70 \%$ ethanol overnight. The fixed cells were treated with $20 \mu \mathrm{g} / \mathrm{ml}$ of PI and $0.2 \mathrm{mg} / \mathrm{ml}$ of RNase A (Thermo Fisher Scientific, CA, USA) for 40 min in dark. After PI staining, cells were resuspended in $500 \mu \mathrm{l}$ of PBS and subjected for flow cytometric analysis by using the BD FACSCalibur platform.

\section{ALDH assay}

ALDH activity was quantified using the Aldefluor Assay Kit (StemCell Technologies Vancouver, Canada) according to the manufacturer's instructions. Briefly, cells were resuspended in Aldefluor assay buffer containing the ALDH substrate bodipy-aminoacetaldehyde for $45 \mathrm{~min}$ at $37^{\circ} \mathrm{C}$. In parallel, equal amount of cells were incubated with diethylaminobenzaldehyde (DEAB), which is an ALDH inhibitor to serve as negative control. Cells were washed with PBS and resuspended in Aldefluor assay buffer before subjected to flow analysis by BD FACSCalibur. ALDH-positive cell population was determined relative to the corresponding $\mathrm{DEAB}$-treated negative control group. The acquired data were analyzed with the FlowJo software 7.6.1 (TreeStar, USA).

\section{Colony-formation assay}

MDA-MB-231 and cisR cells with stable NEAT1 knockdown or empty vector were seeded on 6-well plate at a density of 500 cells/well. After incubation for 7 days, $1 \mathrm{ml}$ of methanol with $1 \%$ crystal violet (Sigma-Aldrich, MO, USA) was added to each well and stained for $30 \mathrm{~min}$. Photographs were taken from each well, and the colonies were counted with the colony-forming unit (CFU) software OpenCFU v3.9.0 (Geissmann) under the same condition.

\section{Clonogenic assay}

The in vitro self-renewal ability of stem cell was reflected by the anchorage-dependent colony formation. Briefly, single cell was seeded in 96-well plate and incubated for 7 days. Single-cell clonogenic ability was evaluated by the number of cell colonies (no less than 50 cells) formed.

\section{Western blotting}

Cells were trypsinized and washed with cold PBS twice and lysed in $100 \mu$ l of lysis buffer (Cell Signaling Technology, MA, USA) for $20 \mathrm{~min}$ on ice. Samples were then centrifuged for $15 \mathrm{~min}$ at $14,000 \times g$ at $4^{\circ} \mathrm{C}$. Protein concentration was determined by the Bradford assay (Bio-Rad, CA, USA). In all, $60 \mu \mathrm{g}$ of protein was loaded into $10-15 \%$ sodium dodecyl sulfate (SDS) gel and fractionated by SDSpolyacrylamide gel electrophoresis and transferred to a polyvinylidene difluoride membrane using a transfer system. The membranes were blocked with $5 \%$ non-fat milk in tris buffered saline with Tween-20 (TBST) to block the unspecific binding sites for $60 \mathrm{~min}$ at room temperature. The membranes were then washed with TBST and incubated with antibodies against cyclin D1 (1:1000), cleaved caspase-3 (1:1000), caspase-3 (1:1000), $\beta$-actin (1:2000), SOX2 (1:1000), and CD44 (1:1000) at $4{ }^{\circ} \mathrm{C}$ overnight with gentle shaking. The membranes were washed with TBST three times and incubated with horseradish peroxidaseconjugated anti-mouse or anti-rabbit antibodies (1:2000) for $60 \mathrm{~min}$ at room temperature. Blots were developed with enhanced chemiluminescence system (Amersham Biosciences, Buckinghamshire, UK) and quantified using ImageJ 1.52a (Wayne Rasband, National Institute of Health).

\section{In vivo xenograft animal model}

The animal experiment ethics (CULATR 4409-17) was approved by the Committee on the Use of Live Animals in Teaching and Research in the University of Hong Kong. Six-week-old female NOD-SCID mice were used in animal models. Briefly, $2 \times 10^{6}$ MDA-MB-231 cells with shNEAT1 or shControl suspended in $100 \mu$ of PBS were injected into mammary fat pad of the mice. Tumor volumes were measured from 2 weeks onwards with palpable mass by using formula [1/2(length $\times$ width $\left.\left.^{2}\right)\right]$ 
every 7 days. In the cisplatin treatment group, cisplatin was administered locally at the tumor mass for 2 weeks after the tumor volume reached $100 \mathrm{~mm}^{3}$ at a dose of 3 $\mathrm{mg} / \mathrm{kg}$ twice a week.

\section{Immunohistochemistry staining}

Briefly, antigen unmasking was performed after deparaffinization. The sections were incubated with primary antibodies (Cell Signaling, MA, USA; Abcam, MA, USA) in recommended diluent overnight at $4{ }^{\circ} \mathrm{C}$. The detection was performed according to the manufacturer's instructions using Signal Stain Boost Detection Reagent (Cell Signaling) and incubated in a humidified chamber for 30 min at room temperature followed by washing. Then Signal Stain DAB was added to each section and subjected to dehydration of the sections by using $95 \%$ and $100 \%$ ethanol, respectively, before mounting.

\section{Statistical analysis}

All experiments were performed in triplicates and data are presented as mean \pm standard deviation (SD). Student's $t$ test and Chi-square test were used as appropriate in calculating the difference between groups. $P$ value $<0.05$ was considered to be statistically significant.

\section{Acknowledgements \\ This study was supported by Dr Ellen Li Charitable Foundation, Kerry Kuok Foundation, Hong Kong Hereditary Breast Cancer Family Registry, and the Seed Funding from Committee on Research and Conference Grants, The University of Hong Kong (201409176192). We thank Mr Chung Wing Bun for assisting in immunostaining.}

\section{Author details}

${ }^{1}$ Department of Surgery, The University of Hong Kong, Pokfulam, Hong Kong. ${ }^{2}$ Department of Medical Oncology, Sir Run Run Shaw Hospital, Medical School of Zhejiang University, Hangzhou, China. ${ }^{3}$ Department of Medical Oncology, Key Laboratory of Biotherapy in Zhejiang, Sir Runrun Shaw Hospital, Medical School of Zhejiang University, Zhejiang, China. ${ }^{4}$ Department of Surgery, The Hong Kong Sanatorium and Hospital, Happy Valley, Hong Kong. ${ }^{5}$ The Hong Kong Hereditary Breast Cancer Family Registry, Shau Kei Wan, Hong Kong

\section{Conflict of interest}

The authors declare that they have no conflict of interest.

\section{Publisher's note}

Springer Nature remains neutral with regard to jurisdictional claims in published maps and institutional affiliations.

Supplementary Information accompanies this paper at (https://doi.org/ 10.1038/s41419-019-1513-5).

Received: 5 November 2018 Revised: 4 March 2019 Accepted: 7 March 2019

Published online: 20 March 2019

\section{References}

1. Djebali, S. et al. Landscape of transcription in human cells. Nature 489, 101 (2012).
2. Hangauer, M. J., Vaughn, I. W. \& McManus, M. T. Pervasive transcription of the human genome produces thousands of previously unidentified long intergenic noncoding RNAs. PLoS Genet. 9, e1003569 (2013).

3. Wright, M. W. \& Bruford, E. A. Naming 'junk': human non-protein coding RNA (ncRNA) gene nomenclature. Hum. Genomics 5, 90-98 (2011).

4. Ma, L., Bajic, V. B. \& Zhang, Z. On the classification of long non-coding RNAs. RNA Biol. 10, 925-933 (2013).

5. Wilusz, J. E., Sunwoo, H. \& Spector, D. L. Long noncoding RNAs: functional surprises from the RNA world. Genes Dev. 23, 1494-1504 (2009).

6. Taft, R. J., Pang, K. C., Mercer, T. R., Dinger, M. \& Mattick, J. S. Non-coding RNAs: regulators of disease. J. Pathol. 220, 126-139 (2010).

7. Schmitt, A. M. \& Chang, H. Y. Long noncoding RNAs in cancer pathways. Cancer Cell 29, 452-463 (2016).

8. Clemson, C. M. et al. An architectural role for a nuclear noncoding RNA: NEAT1 RNA is essential for the structure of paraspeckles. Mol. Cell 33, 717-726 (2009).

9. Sasaki, Y. T., Ideue, T., Sano, M., Mituyama, T. \& Hirose, T. MENepsilon/beta noncoding RNAs are essential for structural integrity of nuclear paraspeckles. Proc. Natl Acad. Sci. USA 106, 2525-2530 (2009).

10. Chakravarty, D. et al. The oestrogen receptor alpha-regulated IncRNA NEAT1 is a critical modulator of prostate cancer. Nat. Commun. 5, 5383 (2014).

11. You, J. et al. MicroRNA-449a inhibits cell growth in lung cancer and regulates long noncoding RNA nuclear enriched abundant transcript 1. Indian J. Cancer 51(Suppl 3), e77-e81 (2014).

12. $\mathrm{Hu}, \mathrm{X}$. et al. The plasma IncRNA acting as fingerprint in non-small-cell lung cancer. Tumour Biol. 37, 3497-3504 (2016).

13. Yuequan, J., Shifeng, C. \& Bing, Z. Prognostic factors and family history for survival of esophageal squamous cell carcinoma patients after surgery. Ann. Thorac. Surg. 90, 908-913 (2010).

14. $\mathrm{Yu}, \mathrm{X} . \& \mathrm{Li}, \mathrm{Z}$. The role of microRNAs expression in laryngeal cancer. Oncotarget 6, 23297-23305 (2015).

15. Li, Y. et al. NEAT expression is associated with tumor recurrence and unfavorable prognosis in colorectal cancer. Oncotarget 6, 27641-27650 (2015).

16. Guo, S. et al. Clinical implication of long non-coding RNA NEAT1 expression in hepatocellular carcinoma patients. Int. J. Clin. Exp. Pathol. 8, 5395-5402 (2015).

17. McCluggage, W. G. Morphological subtypes of ovarian carcinoma: a review with emphasis on new developments and pathogenesis. Pathology 43, 420-432 (2011).

18. He, C., Jiang, B., Ma, J. \& Li, Q. Aberrant NEAT1 expression is associated with clinical outcome in high grade glioma patients. APMIS 124, 169-174 (2016).

19. Adriaens, C. et al. p53 induces formation of NEAT1 IncRNA-containing paraspeckles that modulate replication stress response and chemosensitivity. Nat Med. 22, 861-868 (2016)

20. Mello, S. S. et al. Neat1 is a p53-inducible lincRNA essential for transformation suppression. Genes Dev. 31, 1095-1108 (2017).

21. Idogawa, M., Ohashi, T., Sasaki, Y., Nakase, H. \& Tokino, T. Long non-coding RNA NEAT1 is a transcriptional target of p53 and modulates p53-induced transactivation and tumor-suppressor function. Int. J. Cancer 140, 2785-2791 (2017).

22. Bauer, K. R., Brown, M., Cress, R. D., Parise, C. A. \& Caggiano, V. Descriptive analysis of estrogen receptor (ER)-negative, progesterone receptor (PR)negative, and HER2-negative invasive breast cancer, the so-called triplenegative phenotype: a population-based study from the California Cancer Registry. Cancer 109, 1721-1728 (2007).

23. Choudhry, H. et al. Tumor hypoxia induces nuclear paraspeckle formation through HIF-2alpha dependent transcriptional activation of NEAT1 leading to cancer cell survival. Oncogene 34, 4482-4490 (2015).

24. $\mathrm{Ke}, \mathrm{H}$. et al. NEAT1 is required for survival of breast cancer cells through FUS and miR-548. Gene Regul. Syst. Biol. 10, 11-17 (2016).

25. Jiang, X., Zhou, Y., Sun, A. J. \& Xue, J. L. NEAT1 contributes to breast cancer progression through modulating miR-448 and ZEB1. J. Cell. Physiol. 233 8558-8566 (2018).

26. Li, W. et al. The FOXN3-NEAT1-SIN3A repressor complex promotes progression of hormonally responsive breast cancer. J. Clin. Invest. 127, 3421-3440 (2017).

27. Wang, L. et al. A long non-coding RNA targets microRNA miR-34a to regulate colon cancer stem cell asymmetric division. Elife 5, e14620 (2016).

28. Wang, P. et al. Long noncoding RNA NEAT1 promotes laryngeal squamous cell cancer through regulating miR-107/CDK6 pathway. J. Exp. Clin. Cancer Res. 35, 22 (2016)

29. Wang, X. et al. EGCG enhances cisplatin sensitivity by regulating expression of the copper and cisplatin influx transporter CTR1 in ovary cancer. PLOS ONE 10, e0125402 (2015). 
30. Rinn, J. L. \& Chang, H. Y. Genome regulation by long noncoding RNAs. Annu. Rev. Biochem. 81, 145-166 (2012)

31. Yu, X., Li, Z., Zheng, H., Chan, M. T. \& Wu, W. K. NEAT1: a novel cancer-related long non-coding RNA. Cell Prolif. https://doi.org/10.1111/cpr.12329 (2017).

32. Li, S., Li, J., Chen, C., Zhang, R. \& Wang, K. Pan-cancer analysis of long noncoding RNA NEAT1 in various cancers. Genes Dis. 5, 27-35 (2018).

33. Zhang, M., Wu, W. B., Wang, Z. W. \& Wang, X. H. IncRNA NEAT1 is closely related with progression of breast cancer via promoting proliferation and EMT. Eur. Rev. Med. Pharmacol. Sci. 21, 1020-1026 (2017).

34. Zeng, C. et al. The c-Myc-regulated IncRNA NEAT1 and paraspeckles modulate imatinib-induced apoptosis in CML cells. Mol. Cancer 17, 130 (2018).

35. Gao, C., Zhang, J., Wang, Q. \& Ren, C. Overexpression of IncRNA NEAT1 mitigates multidrug resistance by inhibiting ABCG2 in leukemia. Oncol. Lett. 12, 1051-1057 (2016).

36. Ding, N., Wu, H., Tao, T. \& Peng, E. NEAT1 regulates cell proliferation and apoptosis of ovarian cancer by miR-34a-5p/BCL2. Onco Targets Ther. 10, 4905-4915 (2017).

37. Huang, B. et al. Long non-coding RNA NEAT1 facilitates pancreatic cancer progression through negative modulation of miR-506-3p. Biochem. Biophys. Res. Commun. 482, 828-834 (2017).

38. Li, S., Yang, J., Xia, Y., Fan, Q. \& Yang, K. P. Long noncoding RNA NEAT1 promotes proliferation and invasion via targeting miR-181a-5p in non-small cell lung cancer. Oncol. Res. 26, 289-296 (2018).

39. Fang, L. et al. Long non-coding RNA NEAT1 promotes hepatocellular carcinoma cell proliferation through the regulation of miR-129-5p-VCP-IkappaB. Am. J. Physiol. Gastrointest. Liver Physiol. 313, G150-G156 (2017).

40. Zhen, L., Yun-Hui, L., Hong-Yu, D., Jun, M. \& Yi-Long, Y. Long noncoding RNA NEAT1 promotes glioma pathogenesis by regulating miR-449b-5p/c-Met axis. Tumour Biol. 37, 673-683 (2016).
41. An, J., LV, W. \& Zhang, Y. LncRNA NEAT1 contributes to paclitaxel resistance of ovarian cancer cells by regulating ZEB1 expression via miR-194. Onco. Targets Ther. 10, 5377-5390 (2017).

42. Wang, J. et al. Long non-coding RNA NEAT1 decreases the chemosensitivity of gastric cancer cells via regulating P-glycoprotein expression. Mol. Cell. Toxicol. 13, 317-325 (2017).

43. Tian, X., Zhang, G., Zhao, H., Li, Y. \& Zhu, C. Long non-coding RNA NEAT1 contributes to docetaxel resistance of prostate cancer through inducing RET expression by sponging miR-34a. RSC Adv. 7, 42986-42996 (2017).

44. Liu, F., Tai, Y. \& Ma, J. LncRNA NEAT1/let-7a-5p axis regulates the cisplatin resistance in nasopharyngeal carcinoma by targeting Rsf-1 and modulating the Ras-MAPK pathway. Cancer Biol. Ther. 19, 534-542 (2018).

45. Hu, S. \& Shan, G. LncRNAs in stem cells. Stem Cells Int. 2016, 2681925 (2016).

46. Rosa, A. \& Ballarino, M. Long noncoding RNA regulation of pluripotency. Stem Cells Int. 2016, 1797692 (2016).

47. Hafner, S. J., Talvard, T. G. \& Lund, A. H. Long noncoding RNAs in normal and pathological pluripotency. Semin. Cell. Dev. Biol. 65, 1-10 (2017).

48. Jiang, P. et al. NEAT1 acts as an inducer of cancer stem cell-like phenotypes in NSCLC by inhibiting EGCG-upregulated CTR1. J. Cell. Physiol. 233, 4852-4863 (2018).

49. Gong, W. et al. Knockdown of NEAT1 restrained the malignant progression of glioma stem cells by activating microRNA let-7e. Oncotarget 7, 62208-62223 (2016).

50. LO, P. K. et al. Dysregulation of the BRCA1/long non-coding RNA NEAT1 signaling axis contributes to breast tumorigenesis. Oncotarget $\mathbf{7}$ 65067-65089 (2016). 